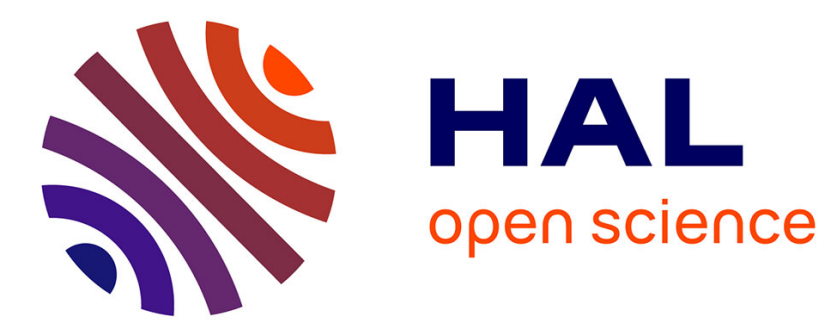

\title{
Yielding and flow of solutions of thermoresponsive surfactant tubes: tuning macroscopic rheology by supramolecular assemblies
}

Anne-Laure Fameau, Arnaud Saint-Jalmes

\section{To cite this version:}

Anne-Laure Fameau, Arnaud Saint-Jalmes. Yielding and flow of solutions of thermoresponsive surfactant tubes: tuning macroscopic rheology by supramolecular assemblies. Soft Matter, 2014, 10 (20), pp.3622-3632. 10.1039/c3sm53001a . hal-00974334

\section{HAL Id: hal-00974334 \\ https://hal.science/hal-00974334}

Submitted on 7 Apr 2014

HAL is a multi-disciplinary open access archive for the deposit and dissemination of scientific research documents, whether they are published or not. The documents may come from teaching and research institutions in France or abroad, or from public or private research centers.
L'archive ouverte pluridisciplinaire HAL, est destinée au dépôt et à la diffusion de documents scientifiques de niveau recherche, publiés ou non, émanant des établissements d'enseignement et de recherche français ou étrangers, des laboratoires publics ou privés. 


\title{
Yielding and flow of solutions of thermoresponsive surfactant tubes: tuning macroscopic rheology by supramolecular assemblies
}

\begin{abstract}
Anne-Laure Fameau*a and Arnaud Saint-Jalmes*b
In this article, we show that stimuli-induced microscopic transformations of self-assembled surfactant structures can be used to tune the macroscopic bulk and interfacial rheological properties. Previously, we had described the formation of micron-sized 12-hydroxystearic acid tubes having a temperaturetunable diameter in the bulk, and also adsorbing at the air-water interface. We report now a detailed study of the bulk and interfacial rheological properties of this solution of thermoresponsive tubes as a function of temperature. In the bulk, the structural modifications of tubes with temperature lead to sharp and non-monotonous changes of rheological behavior. As well, at the air-water interface, the interfacial layer is shifted several times from rigid-like to fluid-like as the temperature is increased, due to morphological changes of the adsorbed interfacial layer. The temperature-induced variations in the fatty acid supramolecular organization and the richness in structural transitions at this microscopic level lead to unique rheological responses in comparison with conventional surfactant systems. Also, this study provides new insights into the required packing conditions for the jamming of anisotropic soft objects and highlights the fact that this system becomes glassy under heating. Due to these unique macroscopic properties both in the bulk and at the interface, this simple system with stimuli-responsive viscoelasticity is of interest for their potential applications in pharmacology or cosmetic formulations.
\end{abstract}

Received 30th November 2013 Accepted 17th February 2014

DOI: 10.1039/c3sm53001a

www.rsc.org/softmatter stimuli such as light and temperature can modify the physical properties of the system on the macroscopic scale, such as the rheological ones. ${ }^{27}$ Solutions of spherical micelles yield low viscosities up to very high surfactant concentrations, whereas the formation of wormlike micelles or vesicles can have strong effects on the rheological properties providing in some cases highly viscous solutions. ${ }^{19}$ For instance, the vesicle-to-wormlike micelle transition induced by heating in the system combining CTAB and 5-methyl salicylic acid leads to a sudden increase of the fluid viscosity. ${ }^{28}$ As well, several studies have demonstrated that with some specific surfactants, UV irradiation leads to a wormlike-to-spherical micelle transition which induces an instantaneous change in viscosity. ${ }^{29-32}$ As another example, structural changes in a DMPG system from gel-state vesicles toward a bilayer network and fluid-state vesicles induce several viscosity transitions with temperature. ${ }^{33}$ However, note that most of the reported systems have a single structural transition induced by an external stimulus, leading to only one rheological change. Also, these studies are mostly based on micelles or vesicles; by comparison, rheological studies of solutions involving self-assembled tubular structures obtained with surfactants remain scarce. ${ }^{34}$

To fabricate responsive materials with various and reversible changes of rheological properties under stimuli, novel surfactant systems with several structural modifications have to be designed. In that spirit, we have recently developed a
${ }^{a}$ UR1268 Biopolymères Interactions Assemblages, Institut National de la Recherche Agronomique, rue de la Géraudière, F-44316 Nantes cedex 3, France. E-mail: anne-laure.fameau@nantes.inra.fr

${ }^{b}$ Institut de Physique de Rennes, UMR CNRS 6251 - Université Rennes 1, Rennes, France. E-mail: arnaud.saint-jalmes@univ-rennes1.fr 
thermoresponsive surfactant system displaying multiple morphological phase transitions. The system is simple and consists of 12-hydroxystearic acid coupled via ion-pairing with ethanolamine as the counter-ion: the composite selfassembles into multilamellar tubes of micron size. ${ }^{26,35}$ The tubes present reversible structural transitions with temperature: (i) the tube diameter is temperature-tunable, it first increases and then decreases within a few degrees upon heating $^{36}$ and (ii) tubes transit into micelles at high temperature. $^{37}$ Moreover, we have shown that the self-assembled tubes can adsorb at the air-water interface, in association with a monolayer, and exhibiting the same temperature tunable structure than in the bulk. ${ }^{38}$ The details of the previous studies on the microscopic structure in the bulk and at the interface are given in Section 3.

The primary objective of the present work is to explore the rheological properties in the bulk and at the interface of this new tubular soft system. The second objective is to determine how these macroscopic properties depend on and reflect the structural transitions of the self-assembled system as a function of the temperature. As described below, it turns out that the bulk viscoelasticity can indeed be widely tuned by the tube morphology and packing fraction i.e. by adjusting the bulk temperature. At the interface, we show that the layer is also non-monotonously tuned from an elastic to a fluid behavior, within only a few degrees, and it is a direct consequence of the structural transitions of the adsorbed layers. We also address issues on how such solutions of surfactant tubes can be glassy under high enough volume fractions, how such systems can develop a temperature-dependant yield stress and how they flow. These yielding and flow features are compared to those of other yield-stress fluids, composed of packed soft colloids (like foams, emulsions, microgels and star polymer glasses).

\section{Materials and methods}

\subsection{Sample preparation}

12-Hydroxystearic acid molecule (Sigma-Aldrich, 99\% purity) was added to ultra-pure water, in order to reach concentrations of 5,10 and $20 \mathrm{~g} \mathrm{~L}^{-1}$. The desired volume of a $1 \mathrm{M}$ stock solution of ethanolamine (Sigma-Aldrich, 99\% purity) is added to reach equivalence (12-hydroxystearic acid/ethanolamine molar ratio $R=1 / 1$ ). The mixture is vigorously vortexed and heated at $80{ }^{\circ} \mathrm{C}$ for $15 \mathrm{~min}$ until all components are dispersed. Prior each measurement, each sample was heated at $80{ }^{\circ} \mathrm{C}$ for $15 \mathrm{~min}$ and cooled at room temperature.

\subsection{Interfacial methods}

The dynamics of the adsorption at the interface is measured by the pendant drop method using a bubble tensiometer. In this method, the surface tension $\sigma$ is calculated by image analysis from the shape of a bubble attached to the tip of the syringe needle using the Laplace equation. The same technique, in oscillatory mode, provides measurements of the interfacial rheological properties. ${ }^{39}$ The interfacial dilatational modulus
$E$ is obtained by performing controlled oscillatory variations of bubble volume. Oscillations are performed $10 \mathrm{~min}$ after drop formation, once the interfaces are in equilibrium. The frequency of the oscillations is varied from 0.005 to $0.01 \mathrm{~Hz}$, and the amplitude is kept constant at 0.05 . For harmonic perturbations of the surface area $A$, the elastic and viscous part of the complex modulus $E$ are derived from the amplitude and phase lag of the surface tension response.

The equilibrium tension and the viscoelastic modulus are determined as a function of the temperature. A thermostatic bath allows the regulation of the temperature inside the quartz cell which is covered during the measurement of aluminum foil in order to limit heat loss. The syringe is not thermostatically controlled but the tip of the needle is situated in the insulated container. A thermal probe placed within the vessel as close as possible to the drop, allows access to the real-time temperature.

\subsection{Bulk rheological methods}

For the bulk rheology, an Anton Paar MCR301 rheometer is used, and experiments are performed in a cone-plate, plateplate and Couette cell setup. As the results are independent of the setup geometry, a largest part of the results presented here has been performed in the cone-plate geometry.

Oscillatory and steady-shear protocols are the two investigated types of measurements. In the oscillatory mode, amplitude- and frequency-sweeps are made, leading to measurements of elastic and viscous moduli, $G^{\prime}$ and $G^{\prime \prime}$, as a function of the strain amplitude $\gamma$ at a constant frequency $f$, and as a function of the frequency $f$ at a constant strain amplitude $\gamma$. In steadyshear measurements, the shear stress $\tau$ and the viscosity $\eta$ are measured as a function of the imposed shear rate $\dot{\gamma}$. For comparisons, some creep tests are also performed (where the applied stress is controlled, and one monitors the induced flow in the sample).

In practice, one has to be careful about the possible slip at the walls, and artifacts linked to high temperatures. First, we selected tools in contact with the samples with roughened walls. Concerning the temperature, the cell is thermalized by the rheometer, and further measurements with an IR camera give us the actual sample temperature. To prevent evaporation, the sample within the cone-plate setup is enclosed in a closed box, and also thermalized using the rheometer. Nevertheless, to be sure to eliminate possible problems linked to evaporation, experiments at high temperature are limited in time. This mostly impacts the steady-shear measurements, and implies a minimal possible shear rate (as discussed below in the text). With these limits, rheological experiments typically require only a few minutes at a given $T$ (especially for the amplitudesweep oscillatory measurements). Consequently, direct observations, after the experiments at any $T$, do not show any sample modifications; as well, the fact that all the rheological data are non-monotonous with $T$ and do not simply always increase with $T$ (as discussed below) also indicates that our observations cannot be simply due to an artifact like evaporation. 


\section{Summary of the previous results on the supramolecular structure in the bulk and at the air-water interface}

We first recall some previous results, mostly obtained from neutron scattering techniques (a small angle and reflectivity setup), from which the morphology of the supramolecular arrangements is deduced. At room temperature, 12-hydroxystearic acid (12-HSA) coupled via ion-pairing with ethanolamine as the counter-ion self-assembles into multilamellar tubes of micron size. ${ }^{35}$ The 12-HSA tubes mean dimensions are $10 \mu \mathrm{m}$ in length $(L)$ and $0.6 \mu \mathrm{m}$ in diameter $(D)$, with some polydispersity. Such 12-HSA tubes exhibit morphological structural transitions with temperature since the outer tube diameter remains first constant with $T$, and starts to rapidly increase at $47^{\circ} \mathrm{C}$, reaching its maximum value around $50{ }^{\circ} \mathrm{C}$. The maximum value of the tube diameter depends on the initial tube length and diameter, but it can reach a few microns for the longest tubes. On average, the diameter is multiplied by 3 at its maximum value, when compared to the minimal value at room temperature. Then, it decreases again upon a further increase of temperature back to the same value than at room temperature $(D \sim 0.6 \mu \mathrm{m})$. Finally, from around $70^{\circ} \mathrm{C}, 12$-HSA tubes melt into micelles. All these structural transitions are completely reversible (Fig. 1). ${ }^{26}$ These previous studies mostly dealt with the structure of the individual supramolecular objects, and did not focus on the concentration of tubes in volume. However, the associated microscopic observations tend to show that the density of tubes is high; we will come back to these issues in Section 6 .

Moreover, in our previous work we demonstrated the ability of these 12-HSA tubes to adsorb at the air-water interface in equilibrium with a fatty acid monolayer (produced by a simultaneous tube unfolding) by using specular neutron reflectivity. ${ }^{38}$ Similarly to 12-HSA tubes in bulk solution, the 12-HSA tubes adsorbed at the interface have a temperature tunable diameter. At $50{ }^{\circ} \mathrm{C}$, the temperature at which in the bulk the 12-HSA tube
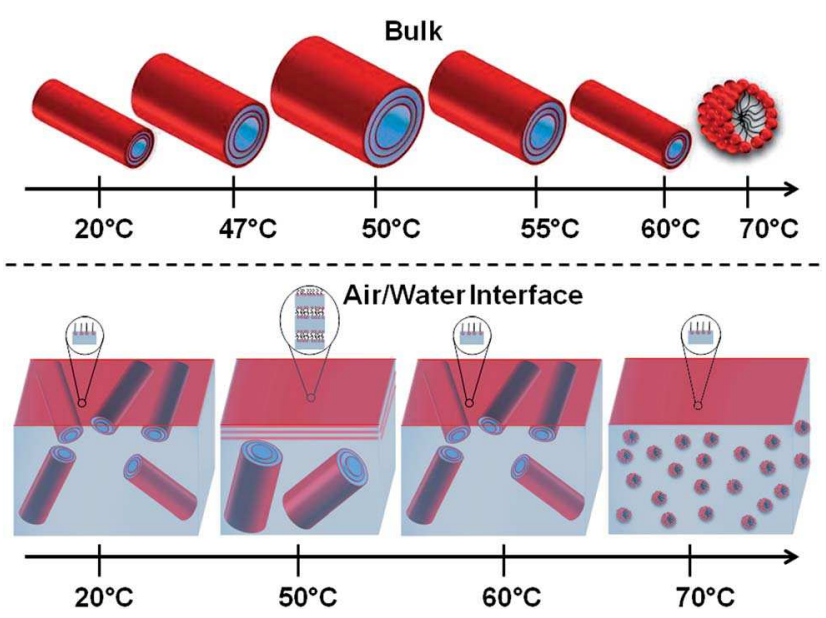

Fig. 1 Sketch summarizing the previous results obtained on the 12HSA-ethanolamine structure in the bulk and at the interface as a function of the temperature. diameter is maximal, a peculiar behavior is deduced by analyzing the scattering data: the 12-HSA tubes at the interface unfold and coalesce to form a multilamellar phase (Fig. 1). Upon further heating, the 12-HSA tubes re-folded and their diameter decreases again, exactly at the same temperature than for 12-HSA tubes in the bulk. At $70{ }^{\circ} \mathrm{C}, 12$-HSA tubes melt into micelles in the bulk and no more tubes stabilize the interface, only monomers are adsorbed. Remarkably, all these structural transitions at the interface are completely reversible as in the bulk (Fig. 1). The 12-HSA-ethanolamine system thus exhibits multiple structural transitions as a function of the temperature both in the bulk and at the air-water interface.

To summarize, the two most important temperature ranges linked to structural transitions are the following: a first one associated with the maximum tube diameter $\left(50^{\circ} \mathrm{C}<T<53^{\circ} \mathrm{C}\right)$, and a second with the transition to the micelles $\left(68{ }^{\circ} \mathrm{C}<T<\right.$ $\left.70{ }^{\circ} \mathrm{C}\right)$. In the new experiments reported below, we will superpose - without any adjusting parameters - these two "microscopic/structural" temperature ranges on the graphs of the "macroscopic/dynamical" data.

\section{Bulk rheology results}

\subsection{Oscillatory measurements}

By sinusoidal oscillatory experiments, one can first monitor how the stress $\tau$ evolves with the amplitude of the applied strain $\gamma$, at a given frequency. In Fig. $2, \tau(\gamma)$ is reported for three temperatures, at $c=10 \mathrm{~g} \mathrm{~L}^{-1}$ and $f=1 \mathrm{~Hz}$. Two regimes are well separated: at low $\gamma, \tau$ varies linearly with $\gamma$, and at higher strains $\tau \sim \gamma^{\delta}$ with $\delta<1$. Such a behavior indicates that the 12-HSA tube solution has first an elastic glassy response, and that it starts to flow above a yield point. These tests provide a direct measurement of the yield stress and strain $-\tau_{y}$ and $\gamma_{y}$ - defined as the coordinates of the point where the two asymptotic regimes cross (Fig. 2). The yield stress $\tau_{\mathrm{y}}$ strongly varies as a function of $T$, whereas $\gamma_{\mathrm{y}}$ remains within the same range of 0.5 to $1.5 \%$ without clear $T$-dependence. The exponent $\delta$ varies between 0.2 and 0.5 with $T$. The evolution of $\tau_{y}$ with $T$ is shown in Fig. 3 , for

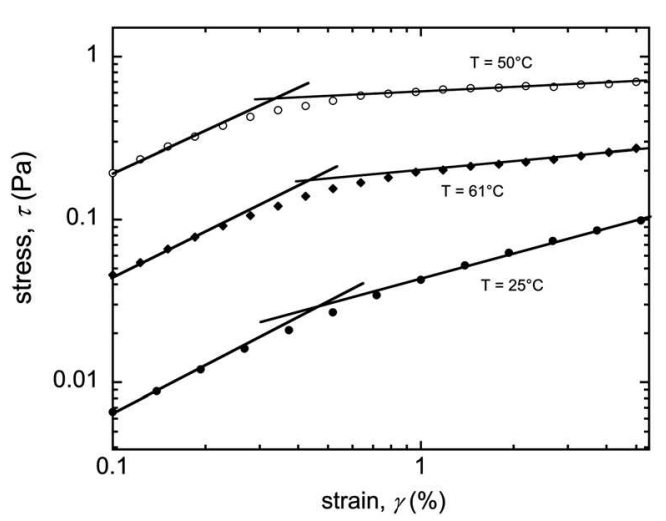

Fig. 2 Shear stress $\tau$ as a function of the strain amplitude $\gamma$ (at constant $f=1 \mathrm{~Hz}$ ) for three different temperatures at a 12-HSA concentration of $10 \mathrm{~g} \mathrm{~L}^{-1}$, evidencing the elastic regime (with a slope of 1 at low $\gamma$ ) followed by the solution yielding. 


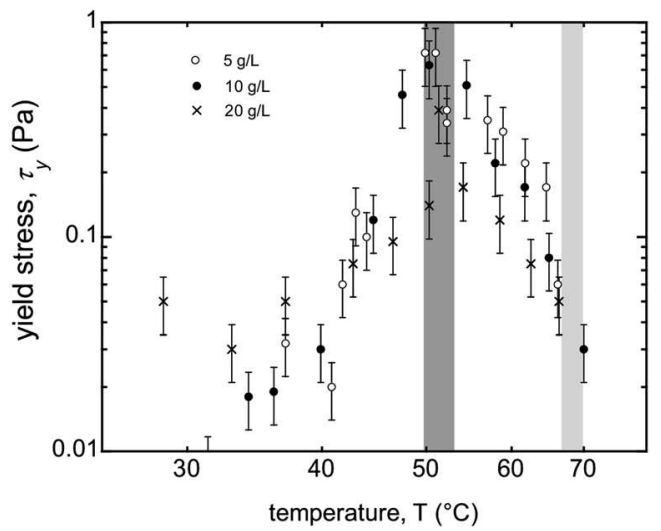

Fig. 3 Evolution of the yield stress $\tau$ with $T$, for three different surfactant concentrations. The vertical grey bars are discussed in the text and correspond to characteristic temperature ranges obtained from structural studies.

three concentrations of surfactants $\left(c=5,10\right.$ and $\left.20 \mathrm{~g} \mathrm{~L}^{-1}\right)$. The same qualitative trend is recovered at all concentrations $c$, with the same non-monotonous behavior, and a maximum in the same range of $T$. For $T>70^{\circ} \mathrm{C}$, the total stress drastically falls to less than $1 \mathrm{~Pa}$, and the elastic regime and the yielding behavior vanish for all $c$. This vanishing of a yielding behavior is also found at the lowest temperatures, but only for $c=5 \mathrm{~g} \mathrm{~L}^{-1}$ : for $T<$ $35{ }^{\circ} \mathrm{C}$, there are no more elastic regimes and yielding.
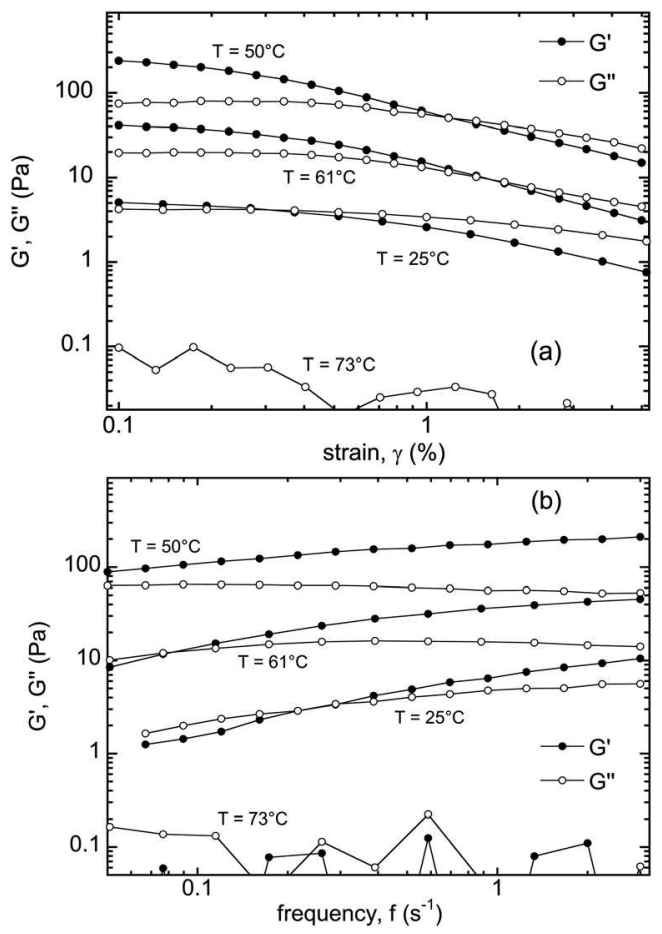

Fig. 4 Results of oscillatory measurements: (a) elastic and viscous moduli plotted as a function of the strain amplitude, $\gamma$ (at constant $f=1$ $\mathrm{Hz}$ ); only four temperatures are selected for clarity. (b) Elastic and viscous moduli plotted as a function of the frequency, $f$ (at constant $\gamma$ $=0.1 \%$.
Secondly, oscillatory tests can be presented in terms of $G^{\prime}$ and $G^{\prime \prime}$, and their evolution with amplitude and frequency. In Fig. 4a, results of amplitude sweeps (at a fixed frequency $f=$ $1 \mathrm{~Hz}$ ), for 12-HSA tubes at a concentration of $10 \mathrm{~g} \mathrm{~L}^{-1}$ and four temperatures are shown. At room temperature $\left(T=25^{\circ} \mathrm{C}\right)$, low values of a few Pa are measured and $G^{\prime} \sim G^{\prime \prime}$. As $T$ increases, the moduli $G^{\prime}$ and $G^{\prime \prime}$ both increase but also separate, leading to a range of low amplitude $\gamma$ where $G^{\prime}>G^{\prime \prime}$, followed by a regime where $G^{\prime \prime}>G^{\prime}$ at higher $\gamma$. This behavior is more and more pronounced up to $T \sim 50-52{ }^{\circ} \mathrm{C}$, as shown in Fig. 4a. At such a temperature, these two regimes can be well identified. For $T>$ $55{ }^{\circ} \mathrm{C}$, the differences between $G^{\prime}$ and $G^{\prime \prime}$ vanishes at room temperature. At the highest $T$, no more elasticity is observed. These results are consistent with the data shown in Fig. 2, the shape of the curve and the balance between the viscoelastic moduli, $G^{\prime}$ and $G^{\prime \prime}$, also confirms a jammed and glassy state at low $\gamma$, followed by yielding of the solution. In such graphs, a usual criterion to define the yielding consists in pointing the start of decrease of $G^{\prime}$. With this definition, the yield occurs for the strain amplitude $\gamma_{\mathrm{y}}$ which does not depend on $T$ : it remains always between $0.5 \%$ and $1.5 \%$, without any significant trend. By contrast, the yield stress varies strongly with $T$, and in quantitative agreement with Fig. 2.

Fig. $4 \mathrm{~b}$ shows how this viscoelastic behavior depends on the frequency, once the amplitude is kept constant $(\gamma=0.1 \%)$. As previously, the moduli strongly depend also on $T$. As $T$ increases, both the moduli $G^{\prime}$ and $G^{\prime \prime}$ first globally increase. There is a high frequency regime where $G^{\prime}>G^{\prime \prime}$, and a low frequency range where $G^{\prime \prime}>G^{\prime}$. The crossover frequency, $f_{c}$, has a non-monotonic behavior: it first decreases strongly with $T$
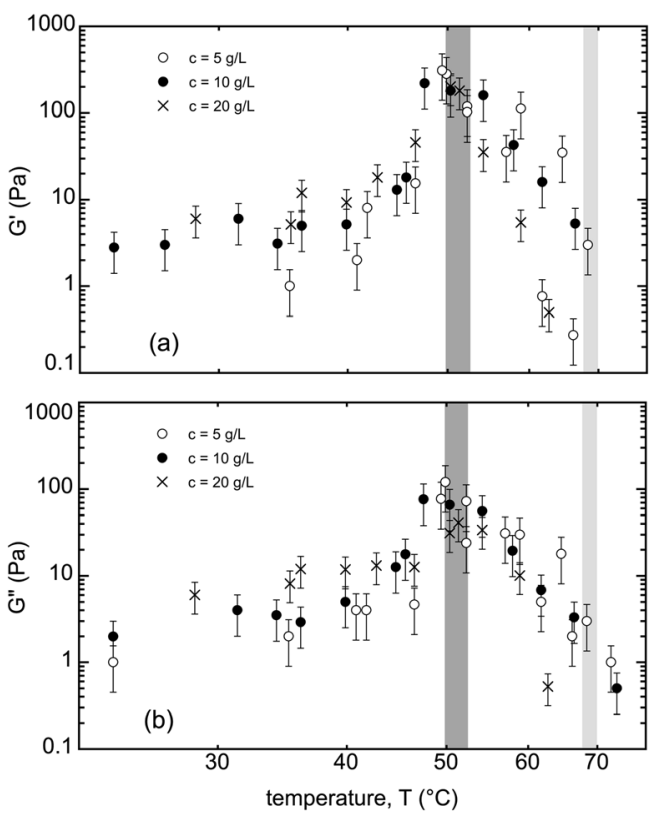

Fig. 5 Bulk viscoelastic moduli as a function of temperature for three concentrations. (a) Elastic modulus as a function of temperature and (b) viscous modulus as a function of temperature. Error bars correspond to the experimental dispersion of the data. The vertical grey bars are discussed in the text and correspond to characteristic temperature ranges obtained from structural studies. 
(down to $f<0.05 \mathrm{~Hz}$ at $T=50{ }^{\circ} \mathrm{C}$ ) and increases at higher temperature. For $T>70{ }^{\circ} \mathrm{C}$, much smaller values are obtained (down to the experimental resolution), with no more elastic contribution. So the glassy regime below yielding only exists above a frequency $f_{\mathrm{c}}$, depending non-monotonously on $T$.

In Fig. 5, the quantitative dependence on temperature can be summarized by focusing on the values of $G^{\prime}$ and $G^{\prime \prime}$ found in the glassy limit, below the yielding, of low amplitude $\left(\gamma<\gamma_{y}\right)$ and high frequencies $\left(f>f_{\mathrm{c}}\right)$, where they do not depend anymore on $\gamma$ and $f$. These values are reported as a function of $T$ in Fig. 5a and $\mathrm{b}$, showing the non-monotonous behavior and the strong moduli variations in only few degrees.

These graphs also include the effect of the concentration: at $c=5 \mathrm{~g} \mathrm{~L}^{-1}$, moduli are close to the experimental resolution and no significant values can be found for $T<35{ }^{\circ} \mathrm{C}$. By contrast, for $T>35{ }^{\circ} \mathrm{C}$, only small differences are observed between the different concentrations, and the highest values are found for the lowest concentration $\left(5 \mathrm{~g} \mathrm{~L}^{-1}\right)$. As previously stated, at $T>70{ }^{\circ} \mathrm{C}$, no more elastic responses are found, for any concentration.

\subsection{Steady-shear measurements}

Fig. 6 shows the flow curves - viscosity vs. shear rate - for different temperatures, of 12-HSA tubes at a concentration of $10 \mathrm{~g} \mathrm{~L}^{-1}$. A shear-thinning behavior is found at all temperatures. The viscosity, $\eta$, also strongly depends on $T$, but again in a nonmonotonous way. To illustrate this behavior, $\eta$ is plotted as a function of $T$ for three selected shear rates in Fig. 7. For $25{ }^{\circ} \mathrm{C}<$ $T<45{ }^{\circ} \mathrm{C}$, the viscosity remains constant. For $T>45{ }^{\circ} \mathrm{C}$, a bump of viscosity is observed. Then, from $T>55^{\circ} \mathrm{C}, \eta$ decreases down towards the lowest measured values (close to pure water viscosity) corresponding to the highest temperatures $\left(T>70{ }^{\circ} \mathrm{C}\right)$. Quantitatively, from the room temperature value, $\eta_{\text {low }}$, to the maximal value, $\eta_{\max }$ (at $T \sim 50{ }^{\circ} \mathrm{C}$ ), the viscosity is multiplied by a factor $x=\eta_{\text {max }} / \eta_{\text {low }}$. This ratio $x$ of the order of 10 becomes independent of $\dot{\gamma}$ at high rates $\left(\dot{\gamma}>0.2 \mathrm{~s}^{-1}\right)$, while it seems to diverge as the shear rate decreases. Note also how the viscosity is divided by orders of magnitude within a few degrees, from $T=65{ }^{\circ} \mathrm{C}$ to $T=70{ }^{\circ} \mathrm{C}$.

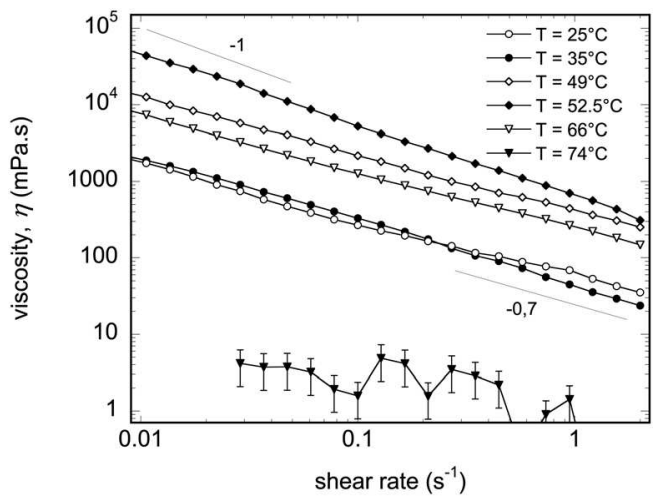

Fig. 6 Steady shear rheometry showing the solution viscosity as a function of the shear rate for various temperatures for a 12-HSA concentration of $10 \mathrm{~g} \mathrm{~L}^{-1}$.

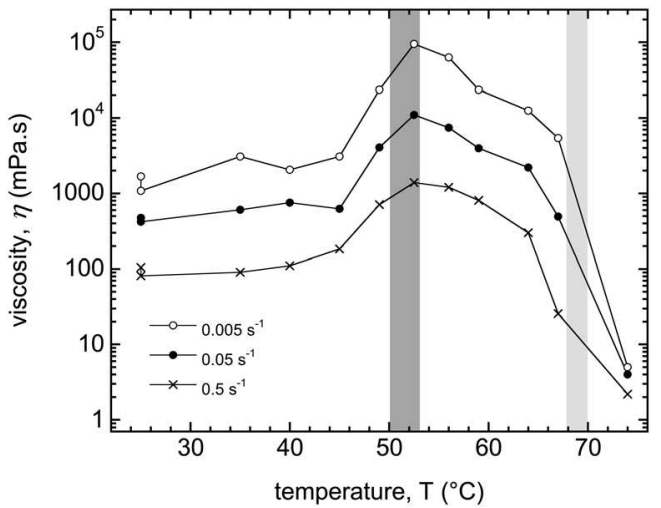

Fig. 7 Viscosity as a function of temperature, for three different shear rates for a 12-HSA concentration of $10 \mathrm{~g} \mathrm{~L}^{-1}$. The vertical grey bars are discussed in the text and correspond to characteristic temperature ranges obtained from structural studies.

In parallel, we can describe the shape of the flow curves $\tau(\dot{\gamma})$ by considering a local shear-thinning exponent, $\tau=\dot{\gamma}^{-\alpha}$, corresponding to the local slope in the log-log plot of Fig. 6. Under the experimental conditions of Fig. 6, one finds that this exponent $\alpha$ remains close to $0.7 \pm 0.05$ over a large range of shear rates and $T$, and that it shifts towards 1 as $T$ increases and $\dot{\gamma}$ decreases (Fig. 6). Further information can be found by varying the concentration and by focusing on the lowest accessible shear rates. However, a measurement point requires an experimental time $t_{\exp }>1 / \dot{\gamma}$. As already introduced in Section 2.3, a minimal shear rate $-\dot{\gamma}=1 \times 10^{-3} \mathrm{~s}^{-1}$ - is selected to avoid too long experimental times and to prevent any sample modification (especially at $T>50{ }^{\circ} \mathrm{C}$ ). Nevertheless, this range remains sufficient to identify two opposite behaviors, which are shown in Fig. 8. The tendency shown in Fig. 6 at $T=52.5^{\circ} \mathrm{C}$, for the low $\dot{\gamma}$ range, remains valid and measurable for $c=10$ and $20 \mathrm{~g} \mathrm{~L}^{-1}$ and for $45^{\circ} \mathrm{C}<T<60{ }^{\circ} \mathrm{C}$ : we always detect some divergence of the viscosity with $\alpha$ approaching 1 , and accordingly the occurrence of a cutoff in stress leading to a plateau value (Fig. 8).
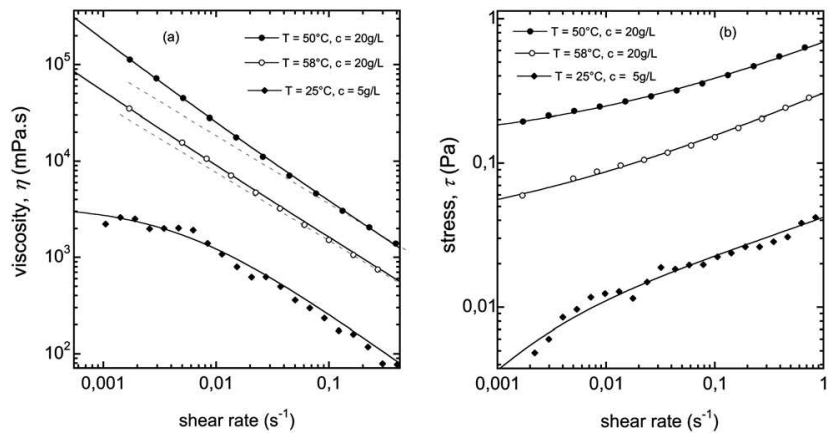

Fig. 8 Shear stress and viscosity at low shear rates, illustrating the two opposite types of behavior as the shear rates tends to zero: (a) viscosity $\eta$ vs. shear rate and (b) stress $\tau$ vs. shear rate; a plateau of viscosity is observed at $\mathrm{C}=5 \mathrm{~g} \mathrm{~L}^{-1}$ and $T=25^{\circ} \mathrm{C}$, while a plateau of stress is found at higher $T$. Solid lines correspond to either Herschel-Bulkley or Cross fits, and the dashed lines are power laws with an exponent -0.7 indicating the scaling behavior at higher shear rates. 
However, comparatively to $T=50{ }^{\circ} \mathrm{C}$, this behavior occurs at smaller $\dot{\gamma}$ as $T$ decreases towards $45{ }^{\circ} \mathrm{C}$ or increases toward $60{ }^{\circ} \mathrm{C}$, with only the beginning of the divergence of viscosity and plateau of stress which can be monitored within the experimental values of $\dot{\gamma}$. This is illustrated by the $60{ }^{\circ} \mathrm{C}$ data shown in Fig. 8 where the dashed line corresponds to the $\dot{\gamma}^{-0.7}$ found at high shear rates, and where only small deviations are measured at $\dot{\gamma}<0.05 \mathrm{~s}^{-1}$. These diverging behaviors are other proofs of a yield stress, in agreement with the oscillatory results. In this limit, data can be fitted by a Herschel-Bulkley model, and quantitative comparisons between the yielding points measured by oscillations or by steady shear will also be done in Section 6. By contrast, an opposite behavior is only well identified in the limit of room temperature and $c=5 \mathrm{~g} \mathrm{~L}^{-1}$, and corresponds to the presence of a plateau of viscosity, and accordingly to a $\tau(\dot{\gamma})$ curve becoming linear (Fig. 8). Data fitting and discussions on the zero-shear viscosity plateau value will also be given in Section 6 .

\section{Interfacial results}

\subsection{Surface tension}

The equilibrium interfacial tension $\sigma$ between air and the 12-HSA solution as a function of the temperature is shown in Fig. 9a. In all cases, the final equilibrium surface tension was obtained in about 10 to 30 seconds. The value remains almost constant for $20{ }^{\circ} \mathrm{C}<T<45{ }^{\circ} \mathrm{C}$. Then at $50{ }^{\circ} \mathrm{C}$, the equilibrium surface tension slightly decreases with a value of $\sigma=22.7 \pm$ $0.2 \mathrm{mN} \mathrm{m}^{-1}$. Upon a further increase of the temperature, for $T>$
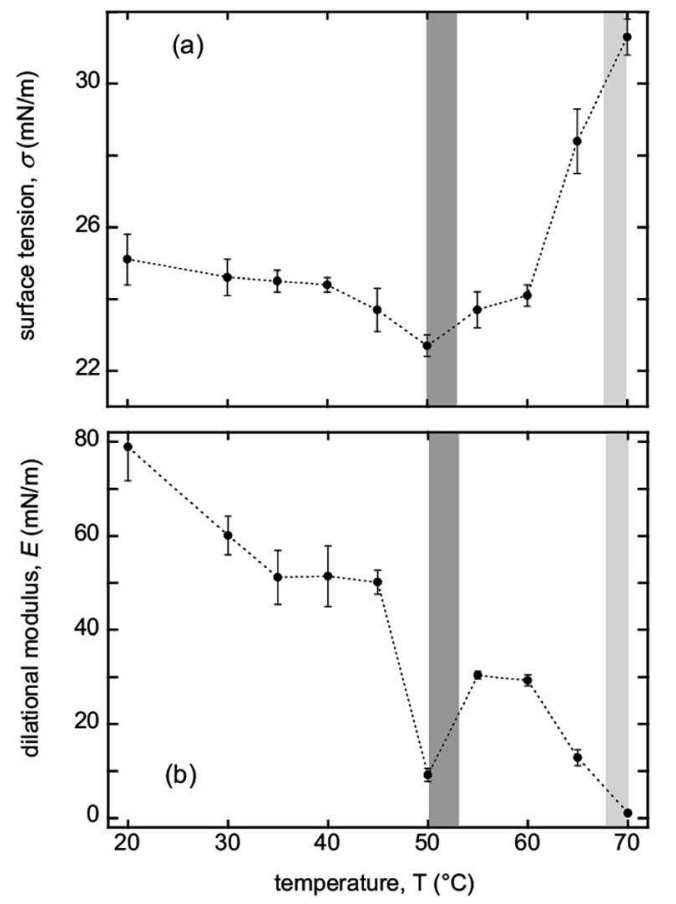

Fig. 9 Interfacial results for $12-\mathrm{HSA}$ solution at $10 \mathrm{~g} \mathrm{~L}^{-1}$ : (a) surface tension and (b) dilatational modulus as a function of the temperature. The vertical grey bars are discussed in the text and correspond to characteristic temperature ranges obtained from structural studies.
$55{ }^{\circ} \mathrm{C}$, the surface tension increases again, back to values obtained for $20{ }^{\circ} \mathrm{C}<T<45{ }^{\circ} \mathrm{C}$. For $T>65^{\circ} \mathrm{C}$, the surface tension increases more abruptly and reaches the maximal measured value $\left(\sigma=31.5 \mathrm{mN} \mathrm{m}^{-1}\right)$ at $70{ }^{\circ} \mathrm{C}$.

It is noted for comparison that in the case of a pure air-water interface, the surface tension decreases linearly with the temperature. At $20^{\circ} \mathrm{C}$, the surface tension is $\sigma=72.8 \mathrm{mN} \mathrm{m}^{-1}$ and at $50{ }^{\circ} \mathrm{C}, \sigma=67.9 \mathrm{mN} \mathrm{m}^{-1} \cdot{ }^{1}$ Thus, in the 12 -HSA system, the thermo-sensitivity in the surface tension comes from the adsorbed layer at the interface.

\subsection{Interfacial dilatational rheology}

On equilibrated interfaces, the viscoelastic dilatational modulus $E$ as a function of the temperature was measured by performing controlled oscillatory variations of bubble volume. Two frequencies of the oscillations are tested 0.005 and 0.01 $\mathrm{Hz}$, but without differences, and the amplitude is kept constant at 0.05 . The results are shown in Fig. 9b, where we report the amplitude of the complex modulus $E$ (the elastic contribution being always much bigger than the viscous one). For $20{ }^{\circ} \mathrm{C}<T<45{ }^{\circ} \mathrm{C}$, this modulus $E$ smoothly decreases, but with values always above $50 \mathrm{mN} \mathrm{m}^{-1}$. Then, for $T \sim 50{ }^{\circ} \mathrm{C}$, it drops abruptly, down to less than $10 \mathrm{mN} \mathrm{m}^{-1}$. For $55{ }^{\circ} \mathrm{C}<T<$ $65{ }^{\circ} \mathrm{C}$, the modulus is measured at values back to $\sim 30 \mathrm{mN} \mathrm{m}^{-1}$, whereas for $T=70^{\circ} \mathrm{C}$, the viscoelastic modulus eventually vanishes.

\section{Discussion}

\subsection{Quantitative correlations between microscopic structures and macroscopic dynamics}

The main issue of this discussion is to understand the origins of the unusual and non-monotonous rheological properties of this 12-HSA-ethanolamine solution in the bulk and at the interface with temperature. Therefore, we compare the previous results obtained on the 12-HSA microscopic structures in tubes and micelles (Fig. 1) with the new macroscopic results (Fig. 3, 5, 7 and 9).

As already explained - shown in Fig. 3, 5, 7 and 9 - two vertical grey bars are added to the new data, and correspond to the two 'microscopic' characteristic temperature ranges: where $D$ is maximum $\left(50{ }^{\circ} \mathrm{C}<T<53{ }^{\circ} \mathrm{C}\right)$ and at the tubemicelle transition $\left(68{ }^{\circ} \mathrm{C}<T<70{ }^{\circ} \mathrm{C}\right)$. In all the macroscopic measurements, it is straightforward to observe that strong correlations between the microscopic and macroscopic measurements exist. All the changes of macroscopic behavior always quantitatively fit with some changes of the microscopic structures. Both types of approaches provide the two same relevant characteristic ranges of $T:\left[50-53{ }^{\circ} \mathrm{C}\right]$ and $\left[68-70{ }^{\circ} \mathrm{C}\right]$. Microscopic and macroscopic properties vary within these same temperature ranges; however, it remains to check and understand if the rheological changes seen on the macroscopic scale are actually consistent with the proposed temperature dependence of the microscopic arrangements (Fig. 1). 


\subsection{Understanding the bulk rheological properties}

In the bulk, as evidenced by the good correlations between microscopic and macroscopic measurements (shown in Fig. 3, 5 and 7), the changes of the supramolecular organization with $T$ are directly transposed to the bulk rheological properties. A major result of both the oscillatory and steady-shear experiments is that this solution containing self-assembled tubes can be considered as a yield stress fluid: below a yielding point, the tubes are mechanically jammed and the system is in a glassy state, while the solution only flows above this yield point. Indeed, the rheological features reported here correspond to what is typically observed for jammed systems obtained at high concentrations of soft and dispersed objects (droplets in emulsions, ${ }^{40}$ bubbles in foams, ${ }^{41-43}$ microgels $^{\mathbf{4 4 , 4 5}}$ or star polymers ${ }^{46-48}$ ).

A first issue is then to determine whether or not these jamming and yielding are consistent with the predicted microstructures. Also, the experimental results indicate that at $c=5 \mathrm{~g}$ $\mathrm{L}^{-1}$ and at room temperature, the solution is not glassy. Another main question is to understand if this frontier between solutions with jammed or unjammed tubes is again consistent with the microscopic picture.

It is known that a solution of fibers or tubes can develop a yield stress, if the fibers/tubes are sufficiently concentrated. ${ }^{\mathbf{4 9 - 5 1}}$ This is different from the jamming of spherical objects, occurring at the random close packing $\left(\phi_{\text {rcp }}=0.64\right),{ }^{52}$ and which implies that the objects must necessarily interpenetrate, overlap or get deformed to go beyond this critical fraction. ${ }^{40-48}$ In fact, the jamming of a solution of non-spherical fiber-like objects occurs at a critical volume fraction, $\phi_{\mathrm{c}}$, well below $\phi_{\mathrm{rcp}}$, and depends on the particle shape. ${ }^{\mathbf{4 9 , 5 3}}$ As $\phi_{\mathrm{c}}<\phi_{\mathrm{rcp}}$, there is still a lot of void in the structure while it is already mechanically jammed. This is originating from the local caging effect, and it requires a minimal decorrelated number of contacts for blocking all motions of a tube (typically 10). ${ }^{49,50}$ As a consequence, the resulting critical volume fraction $\phi_{\mathrm{c}}$ depends on the fiber aspect ratio $L / D$ ( $L$ is the length and $D$ is the diameter), such as $\phi_{\mathrm{c}} \sim$ $5 /(L / D)$ in the limit of $L \gg D$, and $\phi_{\text {c }}$ tends to $\phi_{\text {rcp }}$ as $L / D$ approaches $1 .^{\mathbf{4 9 , 5 0}}$ In our cases, at room temperature, the aspect ratio $\beta=L / D$ is $\beta \sim 20$, providing $\phi_{\mathrm{c}}=0.25$. However, this remains an estimate, as the polydispersity of the tube length $L$ and $D$ must probably be taken into account.

For solutions containing 12-HSA tubes $\left(T<70{ }^{\circ} \mathrm{C}\right)$, the volume fraction of tube in the solution is $\phi=n V_{\text {tube }} / V_{\mathrm{T}}$, where $n$ is the number of tube, $V_{\text {tube }}$ is the tube volume, and $V_{\mathrm{T}}$ is the total volume of the solution. Moreover, increasing the tube diameter $D$ with $T$ (Fig. 1) leads to larger tubes $\left(V_{\text {tube }}=\pi D^{2} L / 4\right)$ and thus to larger $\phi$. However, as the number of monomer remains constant, the length $L$ must also change with $D$. Conserving the number of monomers implies that the total area $A$ of one tube bilayer, $A=\pi D L$ remains constant, so that $D \sim 1 / L$. In that case, $V_{\text {tube }}$ and $\phi$ finally scales as $D$, for a constant $n$ number.

Consequently, based on microscopic information, we can calculate the expected tube fraction at $T=25{ }^{\circ} \mathrm{C}$ for the various concentrations. On the microscopic scale, the area per molecule, $a$, for the HSA in the tube is between 30 and $50 \AA^{2}$ (values corresponding to highly packed or loosely packed monomers), ${ }^{54}$ and we take $t=4$ for the number of bilayers per tube. ${ }^{37}$ We obtain: $\phi\left(T=25{ }^{\circ} \mathrm{C}\right)=0.08,0.16,0.32$, respectively, for 5,10 and $20 \mathrm{~g} \mathrm{~L}^{-1}$ with $a=30 \AA^{2}$, and $\phi\left(T=25^{\circ} \mathrm{C}\right)=0.13$, $0.27,0.53$, respectively, for 5,10 and $20 \mathrm{~g} \mathrm{~L}^{-1}$ with $a=50 \AA^{2}$. These estimations of $\phi$ in the experiments consider monodisperse tubes and constant $n$ number of tubes. ${ }^{37}$

First, we note that these values are qualitatively in agreement with microscopy data of the solution, where the solutions appear crowded with tubes. ${ }^{26,36}$ The most important result is that these predicted values of $\phi$ can be smaller or higher than the theoretical threshold $\phi_{\mathrm{c}}=0.25$. From this theoretical framework, using a single set of microscopic parameters for the three concentrations, it is first expected that the solution at $c=$ $20 \mathrm{~g} \mathrm{~L}^{-1}$ is always sufficiently concentrated to develop a yield stress at all $T$, as $\phi$ is always higher than $\phi_{\mathrm{c}}$. Secondly, it comes that the solution at $c=5 \mathrm{~g} \mathrm{~L}^{-1}$ must not exhibit a yield stress at room temperature, as $\phi\left(T=25{ }^{\circ} \mathrm{C}\right)<\phi_{\text {c }}$. These quantitative predictions correspond to what is observed (Fig. 3). For the intermediate concentration $c=10 \mathrm{~g} \mathrm{~L}^{-1}$, the results are also consistent with the model: the experimental observation of a yield stress, already at $T=25{ }^{\circ} \mathrm{C}$, imply that $a$ is closer to 50 than $30 \AA^{2}$. However, the yield stress is actually so small $(<1 \mathrm{~Pa})$ that $\phi$ is probably in the vicinity of $\phi_{\mathrm{c}}$ at $T=25^{\circ} \mathrm{C}$.

In parallel, in this limit at $c=5 \mathrm{~g} \mathrm{~L}^{-1}$ and $T=25^{\circ} \mathrm{C}$, the flow curve of Fig. 8 provides complementary and consistent information. The stress and viscosity curves can be fitted by a Cross model: ${ }^{55}$

$$
\left(\eta-\eta_{\infty}\right) /\left(\eta_{0}-\eta_{\infty}\right)=1 /\left(1+\zeta \dot{\gamma}^{q}\right)
$$

where $\eta_{0}$ and $\eta_{\infty}$ are the viscosities at the limit of zero and infinite shear rate. It turns out that this model describes well the data, and from the fit shown in Fig. 8 we especially determine a zero-shear viscosity $\eta_{0}=2 \mathrm{~Pa}$ s. Such a value is similar to values found for concentrated dispersions, ${ }^{40}$ at a volume fraction $(\phi=$ $0.55-0.57$ ) just below the jamming (at $\phi_{\text {rcp }}$ in systems of packed spheres). In fact, there are models describing the divergence of the zero-shear viscosity as the volume fraction tends to the critical jamming fraction. The Krieger-Dougherty phenomenological model states that the relative viscosity acts as: ${ }^{56}$

$$
\eta_{\mathrm{r}}=\eta / \eta_{\mathrm{s}}=\left(1-\phi / \phi_{\mathrm{c}}\right)^{-\kappa}
$$

The viscosity $\eta_{\mathrm{s}}$ corresponds to the fluid viscosity in which the tubes are dispersed (here, water, so that $\eta_{\mathrm{s}}=1 \mathrm{mPa} \mathrm{s}$ ) and the exponent $\kappa$ depends on the particle shape and on $\phi_{\mathrm{c}} \cdot{ }^{57}$ For our tubular particle, we use $\kappa=6.5$. As a consequence, a value of $\eta_{0}=2 \mathrm{~Pa} \mathrm{~s}$ implies that the experimental volume fraction is equal to $0.7 \phi_{\mathrm{c}}$, leading to $\phi=0.17$ and in fair agreement with the previous estimations of $\phi\left(T=25{ }^{\circ} \mathrm{C}\right)$ for $c=5 \mathrm{~g} \mathrm{~L}^{-1}$. Therefore, this is confirming that the solution at $c=5 \mathrm{~g} \mathrm{~L}^{-1}$ is below the jamming fraction, and that a small increase in $T$ is sufficient to shift to a glassy state with a yield stress (which is indeed detected at $T=35{ }^{\circ} \mathrm{C}$ ). Altogether and despite the approximations, we are able to delimit quite well under which 
conditions the 12-HSA tube solution is either glassy or not, validating quantitatively the microscopic structure.

Once in the glassy state, regarding the evolution of the yield stress and viscoelastic moduli with $T$, the tube volume fraction increases when $T$ approaches $50{ }^{\circ} \mathrm{C}$ (as $\phi \sim D$ ), meaning that the tubes have to arrange themselves to adjust to this packing increase. Note that this is always possible as this network of tubes is still far from a closely packed organization. The result of such a further packing of the tubes is an increase of the elasticity of the system. As a consequence, it is expected that $\tau_{\mathrm{y}}$ and $G^{\prime}$ increase with $T$ up to the point where $D$ is maximum; this is indeed what is found experimentally (Fig. 3 and 5). To be complete, we must add that as $D$ increases with $T$, the length $L$ is also reduced. This means that the aspect ratio $\beta$ decreases. Therefore, the critical value of packing $\phi_{\mathrm{c}}$ also increases with $T$. But as $\phi_{\mathrm{c}}$ remains bounded by $\phi_{\mathrm{rcp}}$ when $\beta$ goes to 1 , the difference $\left(\phi-\phi_{\mathrm{c}}\right)$ still increases with $T$.

More quantitatively for the variation of $\tau_{\mathrm{y}}$ with $T$, there are predictions for the yield stress, stating that $\tau_{\mathrm{y}} \sim \phi^{3} .^{58,59}$ As $\phi \sim D$, we get $\tau_{\mathrm{y}} \sim D^{3}$. On average, the diameter is multiplied by 3 at its maximum value, when compared to the minimal value at room temperature. This would imply that the yield stress is multiplied by typically $3^{3}$ between their minimal and maximal values. Experimentally, this is consistent with the variation of $\tau_{\mathrm{y}}$ (on the order of 30) found for $c=5$ and $10 \mathrm{~g} \mathrm{~L}^{-1}$ when $T$ increases from 30 to $50{ }^{\circ} \mathrm{C}$ (Fig. 3). However, the experimental increase of $\tau_{\mathrm{y}}$ for $c=20 \mathrm{~g} \mathrm{~L}^{-1}$ is about twice as low as that at 5 or $10 \mathrm{~g} \mathrm{~L}^{-1}$. In fact, the predicted linear dependence of $\phi$ with $D$ would lead to $\phi$ approaching 1 at $50{ }^{\circ} \mathrm{C}$; this shows the limits of our approximations, and we expect that when reaching such high values of packing, the initial tubes must probably rupture and rearrange (leading in particular to a higher $\phi_{\mathrm{c}}$ ). Note also that, as $\gamma_{\mathrm{y}}$ is almost constant, $G^{\prime}$ must vary in the same way as $\tau_{\mathrm{y}}$ with $T$; this is what also what is found (for $c=10 \mathrm{~g} \mathrm{~L}^{-1}, G^{\prime}\left(50^{\circ} \mathrm{C}\right) / G^{\prime}\left(25^{\circ} \mathrm{C}\right) \sim$ 30 ). Lastly, in terms of steady-shear viscosity, for any type of solid dispersion, the fluid viscosity always increases with the concentration of the particles, so with $\phi \sim D$. This is qualitatively consistent with our data showing a maximum viscosity for the larger diameter and packing. Quantitatively, as reported in Section 4 , the viscosity ratio is $x \sim 10$ between 25 and $50{ }^{\circ} \mathrm{C}$ (at stresses well above the yield stress), implying that $\eta$ possibly scales like $D^{2}$.

For further analysis of the solution yielding and flow, comparisons between oscillatory and steady shear measurements can also be made. As explained before, for high enough $c$ and $T$, the available range of shear rates is sufficient to qualitatively detect features corresponding to a yield point (cutoff in stress and divergence of viscosity shown in Fig. 8). Quantitatively, we can try to fit the data by a Herschel-Bulkley form (eqn (3)), where the total stress is the sum of a constant yield stress and a viscous one:

$$
\tau=\tau_{\mathrm{y}}+\xi \dot{\gamma}^{p}
$$

This is the model that is most widely chosen for describing the flow curves of dispersed systems in the glassy state..$^{\mathbf{4 0 , 4 4 , 4 7 , 6 0 , 6 1}}$ As shown in Fig. 8, the stress data can actually be fitted by this model. Accordingly, the viscosity, $\eta=\tau / \dot{\gamma}$, can also be well fitted (Fig. 8b). Adjusting such data allow getting another measurement of the yield stress $\tau_{\mathrm{y}}$, and of the viscous exponent $p$. However, to unambiguously determine $\tau_{\mathrm{y}}$, there must be a significant range of $\dot{\gamma}$ where the viscous term in eqn (3) has to be comparable or small compared to $\tau_{\mathrm{y}}$. Thus, if a yield stress $\tau_{\mathrm{y}}$ becomes low, one must investigate at lower and lower shear rates to be able to detect the signature of this yield stress. Therefore, given our fixed experimental range of shear rates, it is only between 45 and $60{ }^{\circ} \mathrm{C}$ that a signature of the yielding (cutoff in the stress) is observed, because this cutoff yield stress is indeed high enough (Fig. 3). For $T<45{ }^{\circ} \mathrm{C}$ and $T>60{ }^{\circ} \mathrm{C}$, it would require much lower $\dot{\gamma}$ to detect an experimental signature of the yield point. We must also point out that the plateau in stress is not always well defined (Fig. 8), as it is often observed in other systems..$^{40,47,61}$ Thus, even if the data can be fitted, only an apparent value (deduced from a fit) of the yield stress is found, and this method is always much less direct than the oscillatory measurements (where $\tau_{\mathrm{y}}$ is easily observed).

Nevertheless, in the limited range of possible $T$ where it seems reasonable to fit the data, it finally turns out that the values of $\tau_{\mathrm{y}}$ in steady-shear are always smaller, by a factor 1.5-2, than the one extracted from the oscillatory measurements (Fig. 2 and 3). This is again often observed and such a discrepancy illustrates the known difficulties to measure unambiguously a true yield stress from steady-shear measurements. ${ }^{\mathbf{4 0 , 6 2 - 6 4}}$ In fact, the two routes to determine the yield stress do not necessarily rely on the same flow conditions. A first value is obtained by extrapolating the equilibrated and shear-oriented flows at finite shear rates to the limit of $\dot{\gamma}=0$, whereas the second method is based on the occurrence of the first irreversible flow within the initially jammed disordered structure. In practice, it may also be due to the experimental protocol (ramping up vs. ramping down the shear rate), but here we do not find significant differences between such experimental modes. Also, for thixotropic fluids, large variation of the yield stress with the experimental protocol are evidenced, in relation to aging/rejuvenation of the sample, flow heterogeneity (when it is the shear rate which is imposed) and avalanche-like effects. ${ }^{63,64}$ A creep test (applied stress) is considered as a relevant approach to evidence such thixotropic effects, while providing another way of measuring a yield stress. ${ }^{62-65}$ Here, creep experiments are tricky to perform as the yield stress are very low $\left(\tau_{\mathrm{y}}<1 \mathrm{~Pa}\right.$, Fig. 3$)$, and controlled experiments can only be performed when $\tau_{\mathrm{y}}$ is large at high $T$ and $c$. Such tests finally help us to check whether thixotropy is dominant in our system. In fact, we do not observe the features corresponding to thixotropy: there is no significant time-dependence of the viscosity (or shear rate) at controlled stress, nor effects associated with a viscosity bifurcation. ${ }^{63-65}$ Quantitatively, the estimated yield stress (determined as the value of applied stress where some macroscopic flow is induced) falls within the same range as the one obtained from oscillatory measurements. But, as these imposed stresses are small, we cannot have access to an accurate value.

Another important source of discrepancy for the yield stress is that the elastic storage contributions vanish also at low 
frequencies, once $f<f_{\mathrm{c}}$ (i.e. long time scales; Fig. 4b). In these glassy states, this means that there exist intrinsic mechanisms of stress relaxation able to unjam the objects. ${ }^{\mathbf{4 0 , 4 3 , 4 7 , 4 8 , 6 6}}$ The nonmonotonous dependence of $f_{\mathrm{c}}$ with $T$, similar to the one of $\tau_{\mathrm{y}}$, indicates that this is not a thermal effect, but that it depends on the tube volume fraction. Some recombination mechanisms between tubes dominate if the oscillatory forcing is slow enough $\left(f<f_{\mathrm{c}}\right)$, avoiding the storage of elasticity. Otherwise, above $f_{\mathrm{c}}$, the tubes act as solid bodies. Interestingly, the typical $f_{\mathrm{c}}$ falls within an experimentally accessible range of frequency $\left(f_{\mathrm{c}} \sim 10^{-2} \mathrm{~s}^{-1}\right.$, at $\left.T \sim 50-55^{\circ} \mathrm{C}\right)$; this is also the case in some polymer star glasses, ${ }^{47}$ while it is often shifted to extra low values $\left(f_{\mathrm{c}}<10^{-3} \mathrm{~s}^{-1}\right)$ in emulsions or foams. ${ }^{43,55}$ A consequence is that when $\dot{\gamma}$ approaches $f_{\mathrm{c}}$, the system loses its elasticity and progressively corresponds to a viscous liquid. This implies that the stress falls smoothly below the yield stress as $\dot{\gamma}$ decreases below $f_{\mathrm{c}}$, preventing the occurrence of a true plateau and providing a smaller apparent yield stress. This effect, seen also in polymer glasses, ${ }^{47}$ is probably a major reason why the steadyshear measurements underestimate the yield stress. In that respect, the oscillatory mode turns out to be the most reliable route to determine the yield stress.

Lastly, concerning the viscous stress - taken well above the yield stress - it appears to be smaller when extracted from steady shear measurements as compared to the oscillatory ones (Fig. 2), suggesting that the Cox-Merz law may not be valid here. Note though that the comparison can only be made on a small range of oscillatory shear rates $(\dot{\gamma}=\gamma \omega)$, and not well above the yielding. Despite this possible discrepancy, the exponents of the viscous contributions, $\delta$ (Fig. 2) and $p$, are identical, varying between 0.4 and 0.25 , and again similar to others yield stress fluids made of concentrated dispersions. ${ }^{\mathbf{4 0 , 4 4 , 4 5 , 4 7 , 6 0 , 6 1}}$

All together, we are able to understand most of the rheological features and dependence with $T$, and under which conditions there is (or not) a glassy state and a yield stress. We want to point out that all these results are linked to the tube volume fraction and the fact that there is a critical fraction for jamming. All these findings illustrate that this solution of concentrated tubes is a new example of a larger class of concentrated dispersions, as foams, emulsions or pastes, and share the same complex bulk rheology with the same issues ${ }^{62,64}$ (definitions of yielding, flow homogeneity, coupling with intrinsic relaxation mechanisms, etc.). In that spirit, it provides a new interesting candidate to test more complex rheological models, especially because here the dispersed object are no longer isotropic (opposite to droplets, bubbles, microgels or soft polymeric colloids) and have a size tunable by $T$. Further studies are in progress on such issues.

\subsection{Understanding the interfacial rheological properties}

Concerning the interfacial results (Fig. 1b and 9), for the surface tension at equilibrium, we can first note that all the data are in a low range of values (a typical soap surfactant leads to $35 \mathrm{mN}$ $\mathrm{m}^{-1}$ ), meaning that at the air-water interface the 12-HSA monomers are more packed. Together with the rapid adsorption time, this tends to show that the interface is covered first by monomers, most likely coming from the unfolding of the first adsorbed tubes, and then further tubes are adsorbed below this monolayer. The different organization expected between $48{ }^{\circ} \mathrm{C}<$ $T<52{ }^{\circ} \mathrm{C}$ with the formation of a multilayer at the interface has only a small impact on the surface tension. In fact, whatever the temperature $T$ and as long as tubes are present, the top layer at the interface is expected to be made of a dense monolayer of monomers (Fig. 1), leading to a constant interfacial coverage, and then almost independent of the more complex organization below this first monolayer, as it is confirmed by the surface tension measurements. By contrast, the tube-micelle transition occurring in the bulk above $68-70{ }^{\circ} \mathrm{C}$ is clearly recovered in the interfacial tension, providing surface tension values more usual for a soluble small molecular weight surfactant $(30-35 \mathrm{mN}$ $\mathrm{m}^{-1}$ ), where the exchange of monomers between the bulk and interface are possible, as for usual micellar solution. ${ }^{67}$

For dilatational rheology, we found stronger effects of the organization of tubes/monomers adsorbed at the interface, and the dynamical response of the interface is more sensitive to the interfacial structure than the equilibrium surface tension. We first notice the anomalous high elasticity found at room temperature for solution of a priori soluble surfactants: $E$ is around $80 \mathrm{mN} \mathrm{m}^{-1}$, a value usually corresponding to an insoluble interfacial layer. Remarkably, the fatty acid monomers, once freed from the tubular structures and adsorbed at the interfaces, recover their insoluble character, and then practically cannot desorb, leading to a dense insoluble monolayer (in agreement with the low surface tension value). Moreover, tubes are also adsorbed under this monolayer, possibly enhancing the dilational modulus. Comparing Fig. 1b vs. 9b, further links can be made between $E$ and the structural organization at the interface. At $T=50{ }^{\circ} \mathrm{C}$, the expected different interfacial organization, shown in Fig. 1, leads to a more smooth and compressible layer, as $E$ almost vanishes. It is however difficult to separate the role of the multilamellar structure from the fact that less tubes are adsorbed to explain the lower values of $E$. Increasing $T$ to $55^{\circ} \mathrm{C}$ and $60{ }^{\circ} \mathrm{C}$, tubes are expected to be back at the interface with the same microscopic structure and the same tube diameter than between 20 and $45{ }^{\circ} \mathrm{C}$ : indeed, the modulus $E$ is once again high, though lower than at room temperature, maybe due to a solubility increase (still too low to be detected in the equilibrium surface tension). At $T>70{ }^{\circ} \mathrm{C}$, the interfacial layer has lost all its viscoelasticity, as expected for a micellar solution of soluble surfactants able to adjust a constant concentration at the interface, and in agreement with the higher equilibrium value of the surface tension.

Thus, by using the structural modifications of the 12-HSA tubes adsorbed at the interface, it is possible to widely tune within a few degrees - the interfacial viscoelasticity from a "solid-like" behavior characteristic of an irreversibly adsorbed layer to a "fluid-like" behavior of a interface covered by soluble surfactants. For comparison, a PNIPAM layer at the air-water interface is known to exhibit such a transition from "fluid-like" to "solid-like" with temperature. ${ }^{68,69}$ But in that case, there is only one typical temperature separating only two regimes. To the best of our knowledge, this is the first system leading to several consecutive transitions between the "solid-like" interface and the "fluid-like" interface with temperature. 


\section{Conclusions/perspectives}

Bulk and interfacial rheological properties are tuned by the 12-HSA changes of the supramolecular organization in the bulk and at the interface: we show here that the richness in structural transitions of the 12-HSA system is directly transposed to the rheological behavior. In the bulk, a fine tuning of the tube diameter and the transition to micelles with temperature leads to several rheological behaviors. It is striking to find that - for the same aqueous solution containing only simple surfactants and well-suited salts - we can consecutively (and reversibly) shift from a simple shear-thinning fluid to a yield stress fluid and to a low-viscosity Newtonian fluid by increasing the temperature within a relatively small range (from 40 to $70{ }^{\circ} \mathrm{C}$ ). As well, note that the yield stress of the solution actually is multiplied by 30 within less than $10{ }^{\circ} \mathrm{C}$. Similarly, at the airwater interface, the interfacial layer can easily shift from a highly rigid-like to a fluid-like layer in only few degrees due to morphological changes of the adsorbed layer. These rheological responses with temperature are unique in comparison with the conventional low molecular weight surfactant system.

From a fundamental aspect, new results are also obtained to better understand the rheology of concentrated solution of soft tubes. As a consequence of the anisotropic shape of the tube, we show here that a glassy state, where the tubes are jammed, can be obtained if the volume fraction is higher than a critical value $\left(\phi_{\mathrm{c}}=0.25\right)$, which is more than twice smaller than the usual $\phi_{\mathrm{rcp}}$ $=0.64$ (found for isotropic objects). However, despite the differences in the values of the critical fraction, our results show that the jamming, yielding and flow of such a concentrated solution of tubes share many features with those of concentrated solutions of isotropic objects (like emulsions, foams, microgels or star polymer glasses). Thus, our system is original as it provides information on the jamming of objects with a topology different to the usual ones; as well, the originality relies in the fact that we can adjust the packing, either above or below the critical fraction for jamming, by both the surfactant concentration and the temperature. It becomes then possible to investigate if we could reach different glassy configurations depending on the route used to produce a given high tube volume fraction (either from the initial tube concentration or from temperature variations). Note also that, though it has already been observed in some polymeric systems, ${ }^{70}$ it is rather unusual to obtain a transition from a viscous fluid towards a glassy regime by increasing its temperature. Another direction of research would be to study mixtures, either by mixing tubes of different lengths, or by adding other types of soft colloids; in the framework of star polymers, the study of the mixture has indeed provided rich phase diagrams. ${ }^{48,71,72}$

From practical aspects, the structural transition between tubes and micelles with temperature has already been used to produce the first foams exhibiting a temperature-tunable stability. ${ }^{73}$ Moreover, the temperature range for the structural transition can be tuned by the nature of the counter-ion, leading to the design of similar systems for which the various rheological behavior can be adjusted at any given desired temperature. ${ }^{37}$

\section{References}

$1 \mathrm{~J}$. Israelachvili, Intermolecular and surface forces, Academic press limited, London, 1992.

2 T. Shimizu, M. Masuda and H. Minamikawa, Chem. Rev., 2005, 105, 1401.

3 A. Goldsipe and D. Blankschtein, Langmuir, 2006, 22, 3547.

4 C. Morita, Y. Imura, T. Ogawa, H. Kurata and T. Kawai, Langmuir, 2013, 29, 5450.

5 J. Eastoe and A. Vesperinas, Soft Matter, 2005, 1, 338.

6 O. Diat, D. Roux and F. Nallet, J. Phys. II, 1993, 3, 1427.

7 S. Müller, C. Börschig, W. Gronski, C. Schmidt and D. Roux, Langmuir, 1999, 15, 7558.

8 P. M. Mendes, Chem. Soc. Rev., 2008, 37, 2512.

9 P. Brown, C. P. Butts and J. Eastoe, Soft Matter, 2013, 9, 2365.

10 P. Schurtenberger, N. Mazer and W. Känzig, J. Phys. Chem., 1985, 89, 1042.

11 H. Yin, Z. Zhou, J. Huang, R. Zheng and Y. Zhang, Angew. Chem., 2003, 115, 2238.

12 R. Salkar, D. Mukesh, S. Samant and C. Manohar, Langmuir, 1998, 14, 3778.

13 R. Oda, L. Bourdieu and M. Schmutz, J. Phys. Chem. B, 1997, 101, 5913.

14 M. Silvander, G. Karlsson and K. Edwards, J. Colloid Interface Sci., 1996, 179, 104.

15 M. Johnsson, A. Wagenaar and J. B. Engberts, J. Am. Chem. Soc., 2003, 125, 757.

16 P. Hassan, B. Valaulikar, C. Manohar, F. Kern, L. Bourdieu and S. Candau, Langmuir, 1996, 12, 4350.

17 B. F. Silva, E. F. Marques and U. Olsson, Langmuir, 2008, 24, 10746.

18 Z. Lin, J. Cai, L. Scriven and H. Davis, J. Phys. Chem., 1994, 98, 5984.

19 L. M. Walker, Curr. Opin. Colloid Interface Sci., 2001, 6, 451.

20 G. C. Kalur, B. D. Frounfelker, B. H. Cipriano, A. I. Norman and S. R. Raghavan, Langmuir, 2005, 21, 10998.

21 J. Yang, Curr. Opin. Colloid Interface Sci., 2002, 7, 276.

22 D. P. Acharya and H. Kunieda, Adv. Colloid Interface Sci., 2006, 123-126, 401.

23 L. Ziserman, H.-Y. Lee, S. R. Raghavan, A. Mor and D. Danino, J. Am. Chem. Soc., 2011, 133, 2511.

24 V. Soto Tellini, A. Jover, F. Meijide, J. Vázquez Tato, L. Galantini and N. V. Pavel, Adv. Mater., 2007, 19, 1752.

25 L. Galantini, C. Leggio, A. Jover, F. Meijide, N. V. Pavel, V. H. S. Tellini, J. V. Tato, R. Di Leonardo and G. Ruocco, Soft Matter, 2009, 5, 3018.

26 J. P. Douliez, B. Pontoire and C. Gaillard, ChemPhysChem, 2006, 7, 2071.

27 M. Gradzielski, Curr. Opin. Colloid Interface Sci., 2011, 16, 13.

28 T. S. Davies, A. M. Ketner and S. R. Raghavan, J. Am. Chem. Soc., 2006, 128, 6669.

29 R. Kumar, A. M. Ketner and S. R. Raghavan, Langmuir, 2010, 26, 5405.

30 R. Kumar and S. R. Raghavan, Soft Matter, 2009, 5, 797.

31 J. Li, M. Zhao, H. Zhou, H. Gao and L. Zheng, Soft Matter, 2012, 8, 7858 . 
32 A. M. Ketner, R. Kumar, T. S. Davies, P. W. Elder and S. R. Raghavan, J. Am. Chem. Soc., 2007, 129, 1553.

33 M. F. Schneider, D. Marsh, W. Jahn, B. Kloesgen and T. Heimburg, Proc. Natl. Acad. Sci. U. S. A., 1999, 96, 14312.

34 P. Terech and Y. Talmon, Langmuir, 2002, 18, 7240.

35 J. P. Douliez, C. Gaillard, L. Navailles and F. Nallet, Langmuir, 2006, 22, 2942.

36 A. L. Fameau, B. Houinsou-Houssou, B. Novales, L. Navailles, F. Nallet and J. P. Douliez, J. Colloid Interface Sci., 2010, 341, 38.

37 A.-L. Fameau, F. Cousin, L. Navailles, F. Nallet, F. O. Boué and J.-P. Douliez, J. Phys. Chem. B, 2011, 115, 9033.

38 A.-L. Fameau, J.-P. Douliez, F. Boue, F. Ott and F. Cousin, J. Colloid Interface Sci., 2011, 362, 397.

39 G. G. Fuller and J. Vermant, Annu. Rev. Chem. Biomol. Eng., 2012, 3, 519.

40 T. G. Mason, J. Bibtte and D. A. Weitz, J. Colloid Interface Sci., 1996, 179, 439.

41 S. A. Khan, C. A. Schnepper and R. C. Armstrong, J. Rheol., 1988, 32, 69.

42 A. Saint-Jalmes and D. Durian, J. Rheol., 1999, 43, 1411.

43 S. Marze, R. M. Guillermic and A. Saint-Jalmes, Soft Matter, 2009, 5, 1937.

44 M. Cloitre, R. Borrega, F. Monti and L. Leibler, Phys. Rev. Lett., 2003, 90, 068303.

45 S. P. Meeker, R. T. Bonnecaze and M. Cloitre, J. Rheol., 2004, 48(6), 1295.

46 C. N. Likos, H. Löwen, M. Watzlawek, B. Abbas, O. Jucknischke, J. Allgaier and D. Richter, Phys. Rev. Lett., 1998, 80, 4450.

47 B. M. Erwin, M. Cloitre, M. Gauthier and D. Vlassopoulos, Soft Matter, 2010, 6, 2825.

48 D. Truzzolillo, D. Marzi, J. Marakis, B. Capone, M. Camargo, A. Munam, F. Moingeon, M. Gauthier, C. N. Likos and D. Vlassopoulos, Phys. Rev. Lett., 2013, 111, 208301.

49 A. P. Philipse, Langmuir, 1996, 12, 1127.

50 S. Williams and A. Philipse, Phys. Rev. E: Stat. Phys., Plasmas, Fluids, Relat. Interdiscip. Top., 2003, 67, 1301.

51 A. Donev, I. Cisse, D. Sachs, E. A. Variano, F. H. Stillinger, R. Connelly, S. Torquato and P. Chaikin, Science, 2004, 303, 990.

52 J. Bernal and J. Mason, Nature, 1960, 188, 910.
53 A. V. Kyrylyuk and A. P. Philipse, Phys. Status Solidi A, 2011, 208, 2299.

54 K. S. Yim, B. Rahaii and G. G. Fuller, Langmuir, 2002, 18, 6597.

55 M. M. Cross, J. Colloid Sci., 1965, 20, 417.

56 I. M. Krieger, Adv. Colloid Interface Sci., 1972, 3, 111.

57 A. M. Wieranga and A. P. Philipse, Colloids Surf., A, 1998, 137, 355.

58 C. Bennington, R. Kerekes and J. Grace, Can. J. Chem. Eng., 1990, 68, 748.

59 L. H. Switzer III and D. J. Klingenberg, J. Rheol., 2003, 47, 759.

60 N. D. Denkov, V. Subramanian, D. Gurovich and A. Lips, Colloids Surf., A, 2005, 263, 129.

61 S. Marze, D. Langevin and A. Saint-Jalmes, J. Rheol., 2008, 52, 1091.

62 H. A. Barnes, J. Non-Newtonian Fluid Mech., 1999, 81, 133.

63 F. Da Cruz, F. Chevoir, D. Bonn and P. Coussot, Phys. Rev. E: Stat. Phys., Plasmas, Fluids, Relat. Interdiscip. Top., 2002, 66, 051305.

64 P. C. F. Moller, J. Mewis and D. Bonn, Soft Matter, 2006, 2, 274.

65 A. Mujumdar, A. Beris and A. B. Metzner, J. Non-Newtonian Fluid Mech., 2002, 102, 157.

66 A. D. Gopal and D. J. Durian, Phys. Rev. Lett., 2003, 91, 188303.

67 A. Patist, S. Oh, R. Leung and D. Shah, Colloids Surf., A, 2001, 176, 3.

68 R.-M. Guillermic and A. Saint-Jalmes, Soft Matter, 2013, 9, 1344.

69 C. Monteux, R. Mangeret, G. Laibe, E. Freyssingeas, V. Bergeron and G. Fuller, Macromolecules, 2006, 39, 3408.

70 E. Stiakakis, D. Vlassopoulos and J. Roovers, Langmuir, 2003, 19, 6645.

71 C. Mayer, E. Stiakakis, E. Zaccarelli, C. N. Likos, F. Sciortino, P. Tartaglia, H. Löwen and D. Vlassopoulos, Rheol. Acta, 2007, 46, 611 .

72 C. Mayer, F. Sciortino, C. N. Likos, P. Tartaglia, H. Lowen and E. Zaccarelli, Macromolecules, 2009, 42(1), 423.

73 A.-L. Fameau, A. Saint-Jalmes, F. Cousin, B. H. Houssou, B. Novales, L. Navailles, J. Emile, F. Nallet, C. Gaillard, F. Boue and J.-P. Douliez, Angew. Chem., Int. Ed., 2011, 50, 8264. 